\title{
A Robust Polyurethane Depositing System for Overcoming Obstacles in Disaster Scenario Robotics
}

\author{
Alec John Burns ${ }^{1}$, Francesco Ruscio ${ }^{2}$, Sebastiano Fichera ${ }^{1}$ and Paolo Paoletti ${ }^{1}$
}

\begin{abstract}
One of the most difficult challenges faced by ground robots operating in the aftermath of a disaster is the presence of uneven and unstable terrains; in these environments traditional locomotive systems struggle. In this work, a Polyurethane Foam depositing system is proposed to enable ground robots to overcome obstacles and navigate challenging substrates with relative ease. The proposed system is inexpensive, can be added onto existing platforms and enables autonomy via simple control systems. The final mechanical properties of the foam can be tuned in real-time and on board to adapt to different situational requirements. Four deposit foam types have been fully characterized, with volumetric expansion ratios ranging from $20 \times$ to $33 \times$, compressive strengths from $0.16 \mathrm{MPa}$ to $2 \mathrm{MPa}$ and full expansion and set times below 6 minutes in all cases. To show that real-time operations are possible, the system has been implemented on a twotracked rover which was then able to accurately control the amount of deposited foam to form structures such as single and multilayered ramps and blocks. Thanks to this, the vehicle was able to autonomously overcome large objects and chasms that would have otherwise prevented operation.
\end{abstract}

\section{INTRODUCTION}

Disaster scenarios consider the aftermath of an event, such as a sudden accident or a natural catastrophe that causes great damage or loss of life. According to a U.N. report, hundreds of floods, storms, heat waves and droughts have left over 600,000 people dead and 4.1 billion injured or homeless around the world since 1995 [1].

When a disaster strikes, it is critical to find victims and deploy assistance to survivors as quickly as possible. People stranded after an earthquake or hurricane or who are living in a war zone are often stuck for days without food, water or medicines. Infrastructures are usually collapsed in these situations, making it hard for rescuers to reach the afflicted areas to dispense assistance and necessities. Due to this first responders are often exposed to significant risks during the relief efforts [2], often entering highly unstable areas with scarce knowledge of structural integrity of buildings and their interiors.

Aerial, terrestrial and maritime robotic systems can potentially assume a key role mitigating the risks associated with disaster relief, search and rescue and salvage operations [3], while simultaneously ensuring safety of both first responders and survivors. In fact, robots can be deployed quickly in areas deemed too unsafe for humans and can be used to guide rescuers, collect data, deliver essential supplies or provide

\footnotetext{
${ }^{1}$ School Of Engineering, University of Liverpool, Liverpool L69 3GH, UK \{sgaburns, P.Paoletti, sebastiano.fichera\}@ liverpool.ac.uk

2 University of Florence, Firenze 50121, Italy francesco.ruscio@stud.unifi.it
}

TABLE I

COMPARATIVE SUMMARY OF LOCOMOTION SYSTEM PERFORMANCE, TAKEN FROM [8]. LEW = LEGGED WHEELED, LET = LEGGED

TRACKED, WT $=$ WHEELED TRACKED, $\mathrm{L}=\mathrm{LOW}, \mathrm{M}=$ MEDIUM AND $\mathrm{H}=\mathrm{HIGH}$

\begin{tabular}{|l|l|l|l|l|l|l|}
\hline & W & T & Leg & LeW & LeT & WT \\
\hline maximum speed & $\mathrm{H}$ & $\mathrm{M} / \mathrm{H}$ & $\mathrm{L}$ & $\mathrm{M} / \mathrm{H}$ & $\mathrm{M}$ & $\mathrm{M} / \mathrm{H}$ \\
obstacle crossing & $\mathrm{L}$ & $\mathrm{M} / \mathrm{H}$ & $\mathrm{H}$ & $\mathrm{M} / \mathrm{H}$ & $\mathrm{H}$ & $\mathrm{M}$ \\
step climbing & $\mathrm{L}$ & $\mathrm{M}$ & $\mathrm{H}$ & $\mathrm{H}$ & $\mathrm{H}$ & $\mathrm{M}$ \\
slope climbing & $\mathrm{L} / \mathrm{M}$ & $\mathrm{H}$ & $\mathrm{M} / \mathrm{H}$ & $\mathrm{M} / \mathrm{H}$ & $\mathrm{H}$ & $\mathrm{M} / \mathrm{H}$ \\
soft terrain & $\mathrm{L}$ & $\mathrm{H}$ & $\mathrm{L} / \mathrm{M}$ & $\mathrm{L} / \mathrm{M}$ & $\mathrm{M} / \mathrm{H}$ & $\mathrm{H}$ \\
uneven terrain & $\mathrm{L}$ & $\mathrm{M} / \mathrm{H}$ & $\mathrm{H}$ & $\mathrm{H}$ & $\mathrm{H}$ & $\mathrm{M} / \mathrm{H}$ \\
energy efficiency & $\mathrm{H}$ & $\mathrm{M}$ & $\mathrm{L}$ & $\mathrm{M} / \mathrm{H}$ & $\mathrm{M}$ & $\mathrm{M} / \mathrm{H}$ \\
system complexity & $\mathrm{L}$ & $\mathrm{L}$ & $\mathrm{H}$ & $\mathrm{M} / \mathrm{H}$ & $\mathrm{M} / \mathrm{H}$ & $\mathrm{L} / \mathrm{M}$ \\
\hline
\end{tabular}

communication services. Many project have been developed in recent years to tackle some of the most pressing issues [4], [5], [6]. However, taking terrestrial robotic platforms from the often predictable even surfaces of a lab to the disaster zone environments, is hindered by one of their greatest shortfalls: overcoming obstacles.

Several robot architectures have been proposed for driving and climbing on rough terrain, and can be classified into five main categories [7]: single-tracked, multi-tracked, wheeled, quadruped-platforms (or biologically inspired systems) and humanoid. Each class of platforms results in particular benefits, and drawbacks, for overcoming obstacles. Hybrid platforms have been proposed to maximise the pros of their constituent architectures. Such products are often costly and their added benefits limited. A comparison of tracked, wheeled, humanoid and their respective hybrids was performed in [8] and is summarised in Table I for reference. Quadruped and biologically inspired platforms are not included in the table, as these classes represents a very diverse array of systems which are difficult to generalise.

As can be seen from Table I, no platform architecture has so far been proven to outperform the rest. As a result of this, projects have been put forward to utilise material depositing systems with existing odeometry systems to increase performance. One such material used is Polyurethane (PU) and the platform proposed in this paper is a modular PU depositing system to enable ground robots to traverse uneven terrains and overcome obstacles. In section II a brief description of PU foam, its mechanical characteristics and its use in disaster scenario robotics is reported. Section III describes the design for the PU foam depositing module and its integration with a tracked rover. Section IV contains an illustration of the experimental setup used to test the effectiveness of the depositing system, whereas the results 
obtained in these experiments are discussed in Section V. Finally, some final remarks and suggestions for further work are reported in Section VI.

\section{BACKGROUND}

\section{A. Polyurethane Foam}

PU is a synthetic resin composed of polymer units linked by urethane groups. The two part constituents (PU-5800, Polycraft) must be combined with enough vigour for reaction, upon doing so the mix quickly expands and then sets rigid. Expansion typically occurs within $30-50$ seconds and solifidication may take up to 8 minutes. The final mechanical properties of the PU foam are significantly affected by the mix ratio of the two constituent parts, and therefore can be tuned with relative ease. Compressive strengths of over $2 \mathrm{MPa}$ are possible, so that the solidified foam can easily support the weight of a human standing on it. Expansion ratios of over $30 \times$ the original volume are viable, meaning that $25 \mathrm{dm}^{3}$ of solidified foam can be generated from just $0.84 \mathrm{dm}^{3}$ of the two part liquid constituents [9]. These values depend largely on the mixing style and have been recorded through testing on the proposed system, as discussed in section III-B. The foam in its final state is closed-cell, water-proof and lighter than water yet, as mentioned, still strong enough to support the weight of a human climbing thereon. Additionally, these foams adhere to a wide variety of materials including wood, iron, and concrete, among others [9]. Based on these characteristics, this material is suitable for use in disaster scenarios in real-time. Projects have been developed utilising PU foam and amorphous materials to allow robotic platforms to overcome obstacles, see [10], [11], [12] and [13], for example. However, only two projects have utilised a robotic PU foam depositing system for traversing obstacles and are discussed in the section below.

\section{B. Related Work}

The first project using PU foam to overcome obstacles was proposed in [14]. The platform utilises a mechanised syringe to deposit small amounts of two part PU to create a ramp which allowed a small ground robot to traverse an object larger than its original capability. This style of deposit system provides very low mixing rate and thus a very low expansion ratio for the foam. This meant a significant amount of material extrusion was needed to create said ramp. Also, continuous deposition was required if the syringe was to remain unblocked before using all of the material. For the ramp demo shown in this project, multiple syringe cartridges and mixing devices were manually replaced on the system to allow continuous usage. One final remark on this system is that the single rigid nozzle deposit system and small expansion rate resulted in a very complex build requirement, which would be difficult to implement autonomously and was thus manually controlled by a human operator.

The second project related to the using of PU foam to overcome obstacles was proposed in [9]. This platform utilised an aerosol depositing system on a gimbal, with both single part and two part PU tested. This allowed much more flexible deposition than [14], and therefore an autonomous ramping system was possible upon detecting an object. However, the use of aerosol depositing system gives little control over the material being deposited, as the mix ratio and outlet speed are determined by design and cannot be controlled by the system once setup. Also, the use of prepackaged aerosols bring into questions how well this system could be scaled.

The system proposed in this paper tackles the drawbacks highlighted above. It is composed of an on board pumping and mixing system to drive the two part liquids of PU foam to a high rate of reaction, thus giving complete control over the deposition. It also allows both continuous and intermittent operations, as a solvent can be flushed at the end of a deposition phase to prevent blockages.

\section{DESIGN}

This section describes the design of the foam mixing and depositing device, the characterisation of the foam produced by this device and the integration with an autonomous ground tracked vehicle.

\section{A. Deposit System}

Peristaltic pumps are used to drive PU part one and two from their separate reservoirs to a static mixing chamber. This chamber ensures the two parts have been thoroughly mixed without increasing the turbulence to such an extent that the parts begin reacting. This mixing is necessary as multiple outlets may be required (see for example section IIIC), and the viscous nature of the individual parts would otherwise make them flow without mixing. The now combined PU is split across different channels and passed through a static mixing nozzle (MA6.3-21S, Adhesive Dispensing Ltd.) before being ejected at the outlets. A major drawback of systems available in the literature is the blockages that occured between uses, and even during use. This happens as residues, if not treated, will be left in the system and particularly in the static mixing nozzles. As the parts begin to react they become very adhering and as they expand often cause channels to become completely blocked. For the system proposed in this paper a solvent, driven by a third peristaltic pump, is then autonomously flushed through the mixing device at the end of each depositing phase to stop the reaction and eject any residue. This allows the system to be used multiple times without blockage or manual intervention. The whole process is illustrated in Fig. 1.

By driving the system with peristaltic pumps, the amount of liquid being driven at any point is equal to the volume inside the tubing and mixing devices, and is thus independent of the size of the reservoir from which it is being pumped. This implies that the flow generated by the pump is not affected by the size of the reservoirs, unlike in the syringe and aerosol driven designs used in [14] and [9], and therefore the system can potentially be significantly scaled without redesign.

Furthermore, the system can control the flow rate of each pump independently so that the ratio between PU Part one and Part two can be easily controlled. Such ratio controls the 


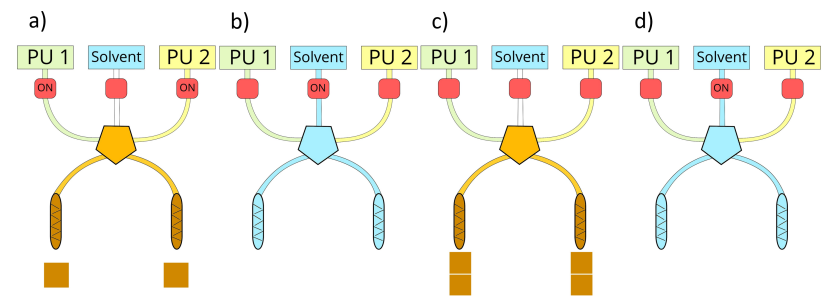

Fig. 1. Illustration of the stages of pumping PU part one and two to create PU foam and the solvent flush stages: a) Pumping of PU part one and two to create first batch of PU foam b) flush of solvent to ensure no blockages after use c) Pumping of PU part one and two to create second batch of PU foam d) flush of solvent. Peristalstic pumps are represented by red symbols, central pentagon represents the mixing chamber and crossed cylinder represent the static mixing nozzles.

properties of the solidified foam, as previously mentioned. For example, if the system required a harder deposit, it could autonomously increase the ratio of PU Part one to the mix. Likewise, increasing the ratio of PU Part two would increase expansion ratio; this could be necessary if maximising the volumetric output was required. Additionally, increasing overall flow velocity increases the turbulence during the mixing of chemicals, thus reducing the time taken to begin expansion. This has the potential to allow the deposited material to be less fluid-like and immediately sticky, with obvious applications for foam deposition on vertical surfaces or gradients. But making the deposit more liquid-like on exit allows the substance to be deposited into crevices and cracks for structural stabilisation. These options would not be possible for syringe or aerosol depositing systems. However, increasing this rate of reaction to a certain level makes the substance more likely to block the static-mixers and thus a maximum overall pump speed is set to prevent this. Finally, the system allows the pumps to drive the liquids to two outlets, although it is possible to increase this number. The importance of this will be mentioned in section III-C.

\section{B. Foam Characterisation}

Four different PU foams obtained via the proposed depositing device are characterised in this section in terms of the most relevant properties: mix ratio, expansion ratio, initial compressive strength, final compressive strength, rise time and set time. Note that the values reported for these four PU foams do not represents the upper and lower limits for properties such as compressive strength and expansion ratio. However, mixes that result in higher expansion ratios result in compressive strengths that are too low for the deposit to be considered useful for the envisaged applications, and vice versa.

Mix ratio considers the volumetric ratio between PU foam part one and part two, and it is controlled via the pump rates of the peristaltic pumps. Expansion ratios were measured by depositing the PU foam in a container and comparing the initial height of the deposited foam with the final height of the deposited foam after expansion had occurred. This method provides conservative estimates of expansion ratios as deposition in free space (e.g. on a surface exposed to

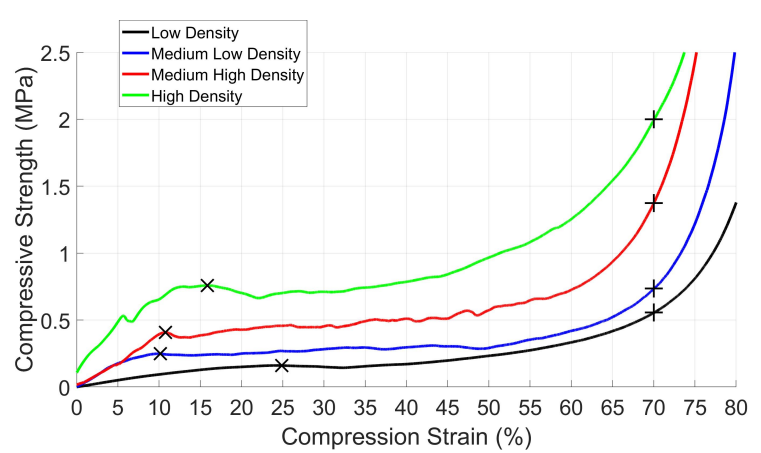

Fig. 2. Stress-strain curves of the foam for different mix ratios, see also Table II.

air) allows more oxidation to occur, and therefore more expansion. However, depositing on a free surface would make it impossible to have consistency due to the different shapes asssumed by the deposit.

Typically, maximum compressive strength considers the amount of force applied per unit area until a material fails, where failure is often defined by the material cracking. However, PU foam, unlikely many solid materials, will continue to deform with sufficient pressure without breakage. Therefore, two alternative definitions of compressive strength are used here: initial compressive strength and final compressive strength. The former is defined as the pressure applied before permanent plastic deformation occurs, and is highlighted with the symbol ' $x$ ' in Fig. 2. Final compressive strength is defined as the pressure at which the height of the deposit is reduced by $70 \%$, as shown in Fig. 2 with the symbol $'+{ }^{\prime}$. Beyond this point the deposit is considered useless for overcoming obstacles. Controlled compression tests were performed by removing a small cube from a free rise deposit and measuring the force and compression/extension using an Instron machine (INSTRON 3345) loading the specimens at $2 \mathrm{~mm} / \mathrm{min}$. The resulting stress-strains curves are shown in Fig. 2 for each of the foams tested.

Rise time is measured from initial deposition until final expansion has occurred. Finally, set time is measured from initial deposition until the foam has fully solidified, this is done by comparing stiffness until it is deemed the material is no longer solidifying and the material is immediately tested in the Instron machine. More importantly than the absolute values of the properties measured for the different PU foams are their relative differences, as they prove that the proposed deposit system can easily control the properties of the deposited material. A summary of properties of the deposited foams are reported in Table II, where each foam is defined by the mix ratio of Part one to Part two.

\section{Robotic Platform}

The proposed PU depositing system has the potential to be combined with any existing robotic platform to extend its ability. For the purposes of testing, the simple low cost ground rover shown in Fig. 3 was used. This platform is a two-tracked vehicle with a track height of $100 \mathrm{~mm}$ and a track length of $300 \mathrm{~mm}$. Being a tracked system provides the 
TABLE II

TABLE 2 . ChARACTERISATION OF FOUR TYPES OF PU FOAM DEPOSITION, WHERE CS STANDS FOR COMPRESSIVE STRENGTH.

\begin{tabular}{|l|l|l|l|l|}
\hline & Low Density & Medium-Low Density & Medium-High Density & High Density \\
\hline Mix Ratio (one:two) & $1: 0.74$ & $1: 1$ & $1: 1.4$ & $1: 1.6$ \\
\hline Expansion Ratio & $33 \times$ & $29 \times$ & $25 \times$ & $20 \times$ \\
\hline Initial Compressive Strength & $0.16 \mathrm{MPa}$ & $0.25 \mathrm{MPa}$ & $0.41 \mathrm{MPa}$ & $0.76 \mathrm{MPa}$ \\
\hline Final Compressive Strength & $0.56 \mathrm{MPa}$ & $0.74 \mathrm{MPa}$ & $1.37 \mathrm{MPa}$ & $2 \mathrm{MPa}$ \\
\hline Rise Time & 37 seconds & 46 seconds & 52 seconds & 55 seconds \\
\hline Set Time & $210-270$ seconds & $240-300$ seconds & $270-340$ seconds & $310-380$ seconds \\
\hline
\end{tabular}

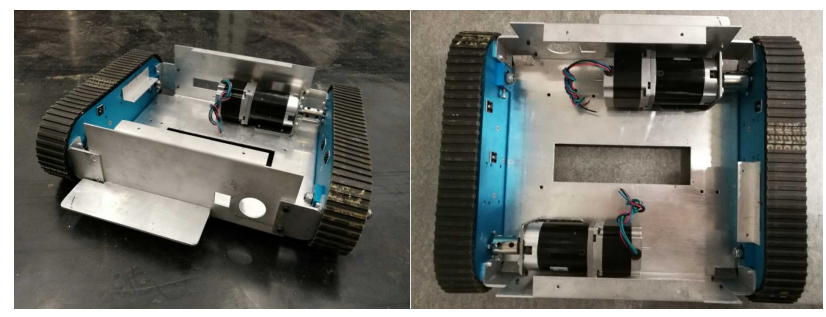

Fig. 3. Images of the rover platform used for testing.

obvious benefit of a high weight distribution when compared to a wheeled vehicle, for example. The rover in question has a pressure value of $0.02 M P a(15 \mathrm{~kg}$ rover on the total surface area of its tracks), making any of the earlier defined foams suitable for the platform. The platform is driven by two large stepper motors (RB-Phi-266, Robotshop), which would allow a $50 \mathrm{~kg}$ payload to be pulled along an even medium friction surface. The rover is driven by a central Arduino Mega 2560 board which controls the motor speeds via two Arduino Nano boards and the pumping systems via another Arduino Mega 2560. A digital compass is connected to the central control board to feed orientation information back to the controller and positional information is calculated from the localisation system discussed in section IV-B. The PU Foam depositing system was mounted on top of the rover with the two outlets positioned directly behind the tracks. As the rover moves, the foam will be deposited, forming two distinct extrusions which are aligned with the rovers tracks. Once the foam has expanded and solidified, the rover can simply climb on said extrusions to increase or maintain altitude. When depositing foam in a straight line, controlling either deposit speed or rover speed allows the platform to create ramp structures as will be seen in section IV. This is an alternative to the complex depositing mechanism proposed in [9] and the complicated ramp structure required in [14].

\section{EXPERIMENTAL SETUP}

Two main experiments are designed to demonstrate the effectiveness of the proposed PU foam depositing system: obstacle climbing and chasm traversing.

\section{A. Sensing and depositing strategies}

Basic ultrasonic distance sensors (HC-SR04) are utilised to determine the presence of obstacles or chasms in front of the vehicle. If an object is detected, a ramp construction procedure is initiated, whereas a void filling function is executed if a chasm is present.

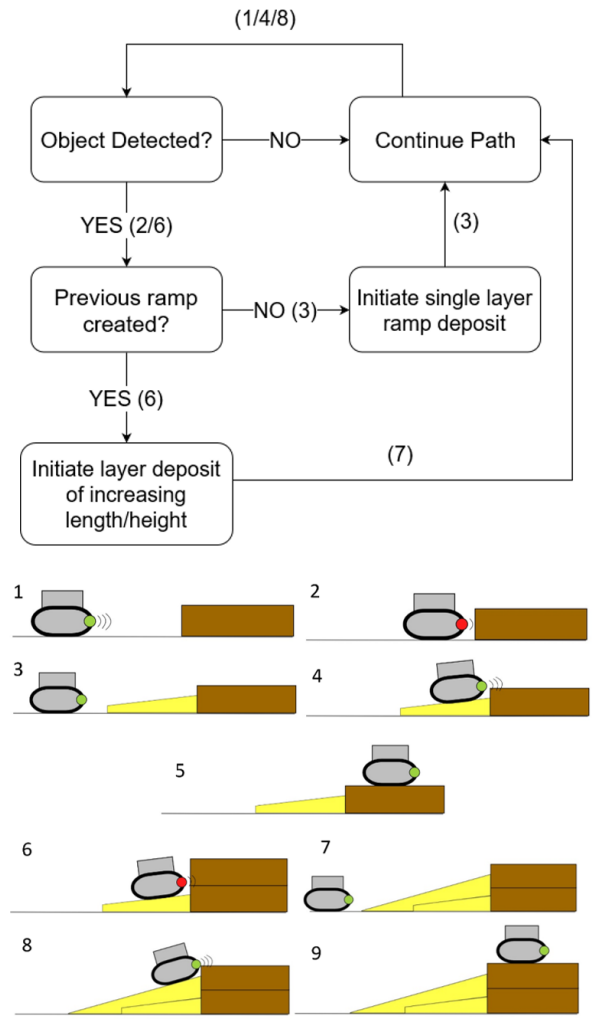

Fig. 4. Flowchart and illustration of the frontal object detection system and ramp building process.

1) Frontal Object Detection: One sensor is placed at the front of the rover, at just above half of the rover track height. It was determined through testing that if an object is detected at this height, the rover will not be able to overcome it independently. As the rover cannot sense if the object is perpendicular to its path, once the object is detected, the rover will begin to move forward at a low motor torque to align the rover front face with the straight edge of an object upon contact. The ramp building protocol, described in the flowchart of Fig. 4 is then initiated, giving rise to the responses illustrated in the same figure.

2) Chasm Detection: The chasm detection scenario considers detecting large gaps in the floor preventing path following. The rover used for testing can overcome chasms of up to $100 \mathrm{~mm}$ (one third of the total length) without falling into said gap, but longer gaps would prevent its motion. To address this challenge, two sensors are placed on the undercarriage of the chassis, facing the ground: one 

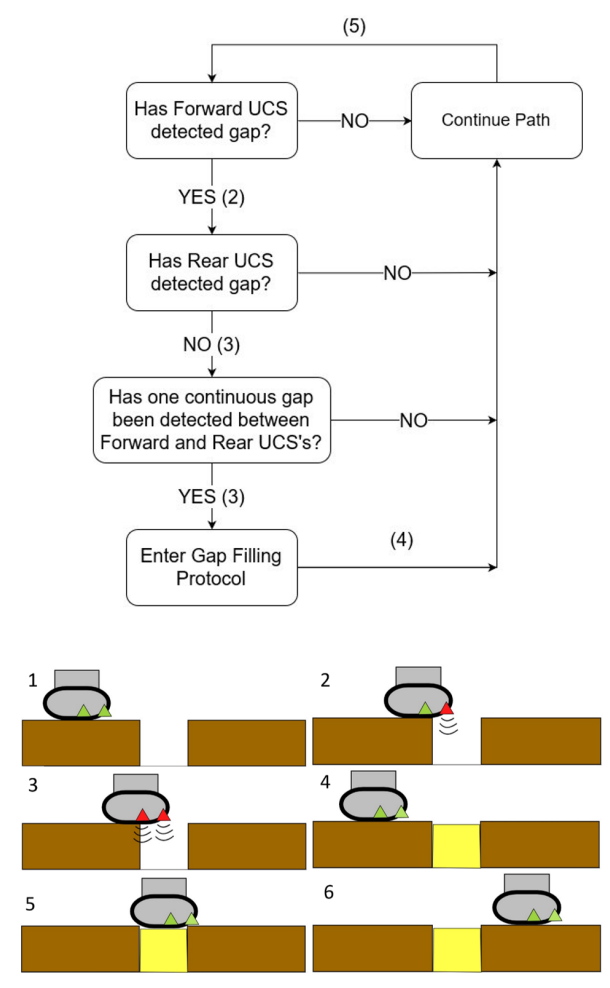

Fig. 5. Flowchart and illustration of the chasm detection system.

is positioned at the front of the rover and other at around one third of the rover length from the front. If both forward and center undercarriage sensors detect a continuous gap, the rover will stop moving and initiate a void filling procedure. At first, the rover uses depth measurements of the chasm to estimates the amount of deposit required. However, if it under deposited (for example if the foam expanded less than expected) then it would once again detect the chasm and repeat the filling procedure. Over-depositing typically leads to foam overflowing the chasm, but the extra amount is usually trivial for the rover to overcome. A flowchart of autonomous response to chasms and respective illustration for the responses are shown in Fig. 5. Chasm detection is overridden when climbing a ramp produced by the system described in IV-A.1.

\section{B. Localisation Platform}

During the experimental tests the rover is tasked with following a desired path within a $4.3 \mathrm{~m}$-by- $3.1 \mathrm{~m}$ arena and the obstacle avoidance protocols described above activate if said path is being blocked. To perform path following, a low-cost localisation system based on ultrasonic sensing and time difference of arrival was designed, taking inspiration from [15] and [16]. The compact ultrasound emitter shown in Fig. 6 was designed to generate omnidirectional train of ultrasound pulses which are then picked up by several fixed receivers measuring the time difference of arrival. A least squares approach is used to analytically obtain a first estimate of the emitter position, which is then refined through steepest descent optimisation. All processing is done via a standard

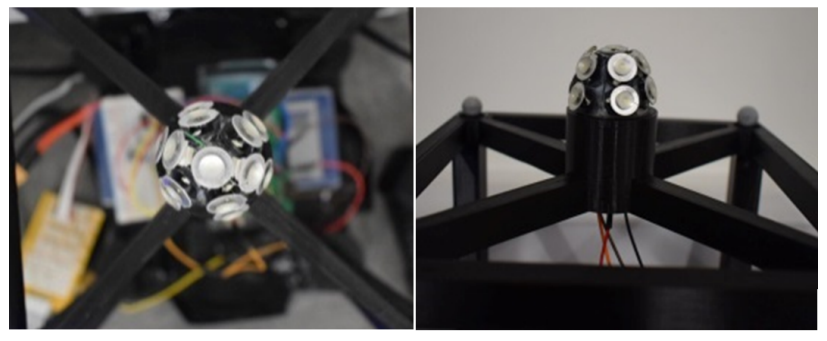

Fig. 6. The omnidirectional ultrasonic emitter designed for the localisation system.

Arduino platform, proving the low computational demands of the method. Localisation results have been validated against a state-of-the-art Optitrack motion capture system composed of 8 Prime $17 \mathrm{~W}$ cameras. The ultrasound localisation system allows estimation of rover position within an accuracy of less than $3 \mathrm{~cm}$ over $89 \%$ of arena and less than $1 \mathrm{~cm}$ over $43 \%$ of the arena. Overall, the mean error localisation error is $1.57 \mathrm{~cm}$ and the average standard deviation is $1.39 \mathrm{~cm}$ throughout the arena, making it suitable for being embedded on the mobile robotic platform used for the experiments.

\section{RESUlts}

Three experiments were carried out with both detection systems being operational. The rover is given a straight line path to follow, but if any object is detected along this path the vehicle must work out how best to overcome them. All three experiments require the ability to: i) detect an obstacle that prevents the rover from following the planned path ii) eject the PU foam correctly iii) flush the system to ensure no blockages occur iv) wait until the foam has cured and then overcome obstacle using the deposited foam. The first two experiments consider frontal obstacles and the third considers chasm detection. For all three tests the mix ratio of PU Part one:Part two was fixed at $1: 1$ (Medium-Low Density foam) so that it can settle within 6 minutes, expand around $29 \times$ and have sufficient strength to support the rover weight. All the obstacles were tested at first to ensure that the rover could not overcome them without using the PU depositing system: the vehicle was not able to grip onto the material for the frontal objects and got stuck in the chasm.

\section{A. Small Frontal Object Test}

In the first experiment, a $60 \mathrm{~mm}$ high block - $60 \%$ of the $100 \mathrm{~mm}$ rover height - was placed along the desired path. The rover detected the object, aligned itself and began the the ramp deposit procedure. The vehicle created the ramp by varying pump speed as it moved away at a constant speed so that more material was deposited closer to the object, as shown in Fig. 7. The platform then waited for the foam to expand and solidify before using the deposit to continue its path. No further obstacle was detected and the rover could successfully climb onto the object. The total time to run this experiment was 6 minutes and 42 seconds. 


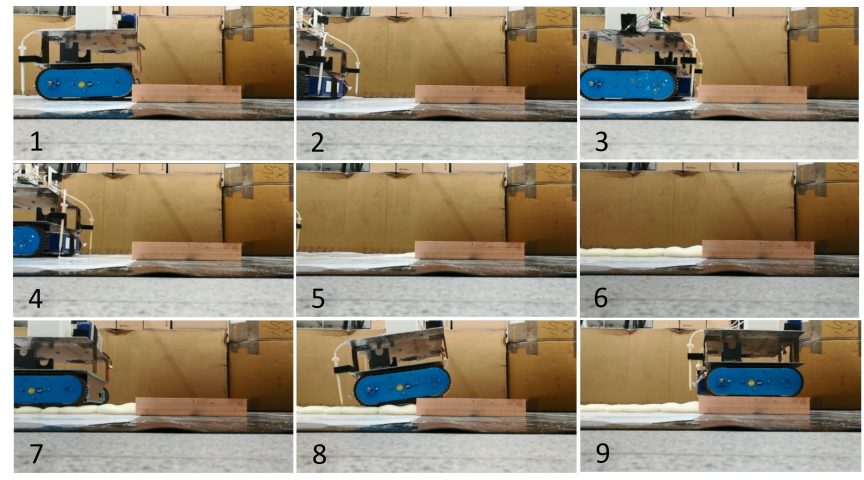

Fig. 7. Small object test: the stages of the rover detecting a $60 \mathrm{~mm}$ high block and depositing a ramp to climb onto the obstacle.

\section{B. Large Frontal Object Test}

In the second experiment a $130 \mathrm{~mm}$ high block - $130 \%$ times the rover height - was placed along the planned path. The rover detected the object and conducted the same first layer ramp deposit procedure as in the previous experiment. However, upon climbing the ramp it detects the object again. Knowing it has previously deposited a ramp, the rover initiates the ramping procedure but deposits foam for an increased distance compared to the previously created ramps. The platform then waited for the second layer to cure and was able to overcome the object, as shown in Fig. 8. The success of this test proves that building large, multi-layered ramp structures are possible and that the system ensures no blockages occur between layers/uses. Total time for this experiment was 13 minutes and 42 seconds.

\section{Chasm Test}

In the final experiment a $160 \mathrm{~mm}$ long chasm was placed along the rover's path, over half the $300 \mathrm{~mm}$ rover tracks length. The chasm was $80 \mathrm{~mm}$ deep and $400 \mathrm{~mm}$ wide. When the rover detected a small gap with the frontal undercarriage sensor, it reduced its speed to ensure it had sufficient time to either detect whether it was able or not to overcome the chasm without depositing material. Once the rover detected that the chasm was too long by using both undercarriage sensors, it started its gap filling procedure. The material depositing system estimated the amount of material to be deposited from the knowledge of the depth of the chasm (measured by sensors), performed the deposit and then waited for this to expand and solidify. The rover successfully filled the chasm and traversed the gap as shown in Fig. 9. Total time for this experiment time was 5 minutes and 60 seconds.

\section{Summary of experimental results}

A summary of the experimental results is reported in Table III, showing that the proposed PU foam depositing system enables the rover to overcome obstacles which were previously insurmountable. In all cases, the volumetric expansion ratio was between $29 \times$ and $32 \times$, showing the robust control over the mixing process and, hence, the final mechanical properties of the foam.
TABLE III

TABle 3 . Summary of EXPERIMENTAL RESUlts, WHERE H=HEIGHT, $\mathrm{D}=$ DEPTH, $\mathrm{L}=$ LENGTH AND VOL=VOLUME

\begin{tabular}{|l|l|l|l|}
\hline & Test One & Test Two & Test Three \\
\hline Type & Small Frontal & Large Frontal & Chasm \\
\hline Dimensions & H: $60 \mathrm{~mm}$ & $\mathrm{H}: 130 \mathrm{~mm}^{\mathrm{m}}$ & DxL:100x200 $\mathrm{mm}$ \\
\hline Deposit Vol & $2000 \mathrm{~cm}^{3}$ & $5000 \mathrm{~cm}^{3}$ & $4000 \mathrm{~cm}^{3}$ \\
\hline PU used & $63^{3}$ & $170 \mathrm{~cm}^{3}$ & $126 \mathrm{~cm}^{3}$ \\
\hline Run Time & 6 mins42secs & $13 \mathrm{mins} 42$ secs & 5 mins60secs \\
\hline
\end{tabular}

\section{Vi. Final Remarks and Future Work}

In this paper an inexpensive and easy-to-use PU foam depositing system is proposed. The device is designed as an independent module for existing robotic platforms to expand their capabilities. Thanks to its design, it can be utilised without complicated control algorithms to allow ground vehicles to autonomously overcome obstacles. This system allows complete control over the deposited material: the PU foams expansion ratio and final compressive strength can be tuned autonomously according to the situational requirement. The embedded flush system allows the long term use of the module without blockage, a typical drawback of existing platforms. Initial tests show that the proposed device provides a significant improvement of the capability of ground vehicles to move on uneven terrains. The proposed device then removes the main obstacle for using ground robots in disaster scenarios. Future work will focus on the use of the system with intelligent platforms, so that different foam properties are utilised autonomously for varying situations. Collaborative robots for large scale efforts are also being considered.

\section{ACKNOWLEDGMENT}

This research was supported by Apadana Management 3 Ltd. The authors also wish to thank colleagues from the University of Liverpool who provided insight and expertise that greatly assisted the research.

\section{REFERENCES}

[1] T. C. for Research on Epidemiology of Distasters (CRED) and T. U. N. O. for Disaster Risk Reduction, "The human cost of weather related disasters 1995-2015," 2016. [Online]. Available: https://www.preventionweb.net/files/46796_cop21 weatherdisastersreport2015.pdf

[2] D. A. Alexander and S. Klein, "First responders after disasters: A review of stress reactions, at-risk, vulnerability, and resilience factors," Prehospital and Disaster Medicine, vol. 24, no. 2, p. 87-94, 2009.

[3] R. R. Murphy, S. Tadokoro, D. Nardi, A. Jacoff, P. Fiorini, H. Choset, and A. M. Erkmen, Search and Rescue Robotics. Berlin, Heidelberg: Springer Berlin Heidelberg, 2008, pp. 1151-1173.

[4] K. Nagatani, S. Kiribayashi, Y. Okada, S. Tadokoro, T. Nishimura, T. Yoshida, E. Koyanagi, and Y. Hada, "Redesign of rescue mobile robot quince," in 2011 IEEE International Symposium on Safety, Security, and Rescue Robotics, Nov 2011, pp. 13-18.

[5] R. R. Murphy, J. Kravitz, S. L. Stover, and R. Shoureshi, "Mobile robots in mine rescue and recovery," IEEE Robotics Automation Magazine, vol. 16, no. 2, pp. 91-103, June 2009.

[6] F. Matsuno and S. Tadokoro, "Rescue robots and systems in japan," in 2004 IEEE International Conference on Robotics and Biomimetics, Aug 2004, pp. 12-20.

[7] M. Brunner, B. Brüggemann, and D. Schulz, "Towards autonomously traversing complex obstacles with mobile robots with adjustable chassis," in Proceedings of the 2012 13th International Carpathian Control Conference, ICCC 2012, 052012. 

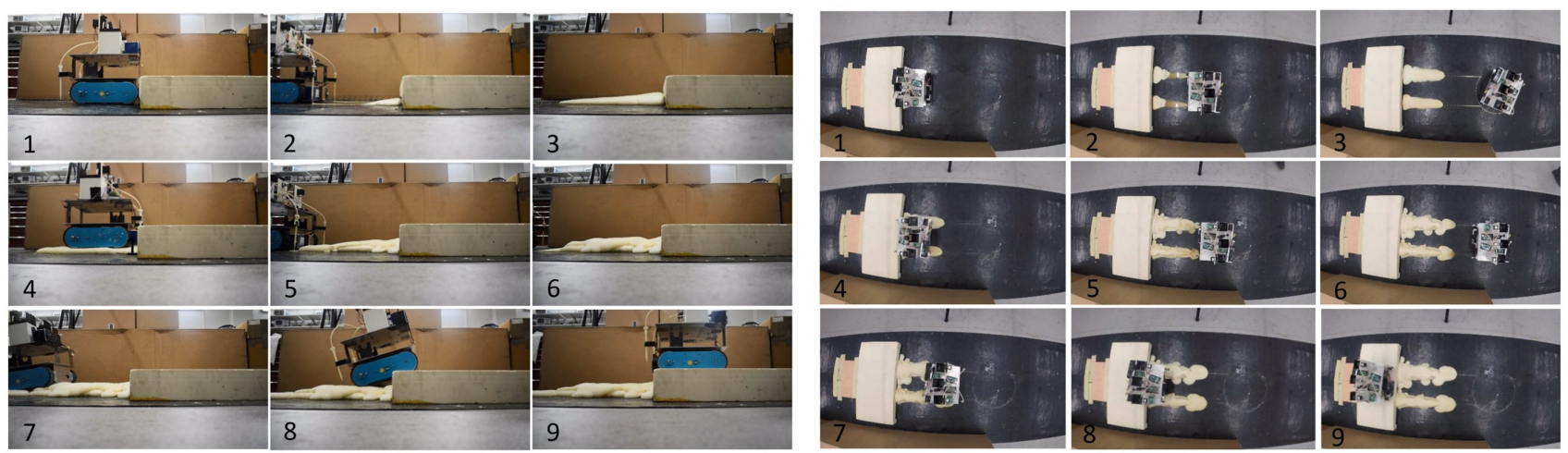

Fig. 8. Large obstacle test: the stages of the rover detecting a $130 \mathrm{~mm}$ high block and depositing a ramp foam to climb onto the obstacle.

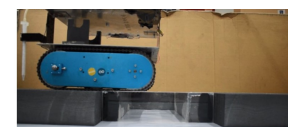

1
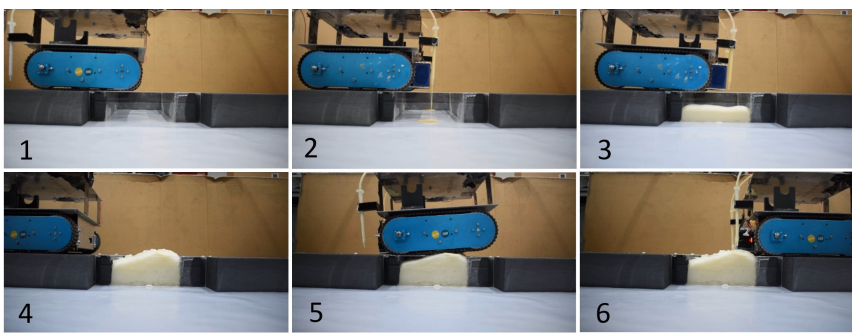

Fig. 9. Chasm test: the stages of the rover detecting a $160 \mathrm{~mm}$ long chasm and depositing PU foam to fill the gap and traverse the chasm.

[8] L. Bruzzone and G. Quaglia, "Review article: locomotion systems for ground mobile robots in unstructured environments," Mechanical Sciences, vol. 3, pp. 49-62, 072012.

[9] R. Fujisawa, N. Nagaya, S. Okazaki, R. Sato, Y. Ikemoto, and S. Dobata, "Active modification of the environment by a robot with construction abilities," ROBOMECH Journal, vol. 2, no. 1, p. 9, Apr 2015.

[10] N. Napp and R. Nagpal, "Distributed amorphous ramp construction in unstructured environments," in Distributed Autonomous Robotic Systems, M. Ani Hsieh and G. Chirikjian, Eds. Berlin, Heidelberg: Springer Berlin Heidelberg, 2014, pp. 105-119.

[11] G. Hunt, F. Mitzalis, T. Alhinai, P. A. Hooper, and M. Kovac, "3d printing with flying robots," in 2014 IEEE International Conference on Robotics and Automation (ICRA), May 2014, pp. 4493-4499.

[12] H. Ardiny, S. Witwicki, and F. Mondada, "Construction automation with autonomous mobile robots: A review," in $20153 \mathrm{rd}$ RSI International Conference on Robotics and Mechatronics (ICROM), Oct 2015, pp. 418-424.

[13] N. Napp and R. Nagpal, "Robotic construction of arbitrary shapes with amorphous materials," in 2014 IEEE International Conference on Robotics and Automation (ICRA), May 2014, pp. 438-444.

[14] N. Napp, O. R. Rappoli, J. M. Wu, and R. Nagpal, "Materials and mechanisms for amorphous robotic construction," in 2012 IEEE/RSJ International Conference on Intelligent Robots and Systems, Oct 2012, pp. 4879-4885.

[15] U. Yayan, H. Yucel, and A. Yazıcı, "A low cost ultrasonic based positioning system for the indoor navigation of mobile robots," Journal of Intelligent \& Robotic Systems, vol. 78, no. 3, pp. 541-552, Jun 2015. [Online]. Available: https://doi.org/10.1007/s10846-0140060-7

[16] K. Cheung, H. So, W.-K. Ma, and Y. Chan, "A constrained least squares approach to mobile positioning: Algorithms and optimality," EURASIP Journal on Advances in Signal Processing, vol. 2006, no. 1, p. 020858, Dec 2006. [Online]. Available: https://doi.org/10.1155/ASP/2006/20858 